\title{
Aging reduces experience-induced sensorimotor plasticity. A magnetoencephalographic study
}

\author{
Alison Mary ${ }^{\mathrm{a}, \mathrm{c}, *}$, Mathieu Bourguignon ${ }^{\mathrm{b}, \mathrm{c}, \mathrm{d}}$, Vincent Wens ${ }^{\mathrm{b}, \mathrm{c}}$, Marc Op de Beeck ${ }^{\mathrm{b}, \mathrm{c}}$, Rachel Leproult ${ }^{\mathrm{a}, \mathrm{c}}$, \\ Xavier De Tiège ${ }^{\mathrm{b}, \mathrm{c}}$, Philippe Peigneux ${ }^{\mathrm{a}, \mathrm{c}, *}$ \\ a UR2NF - Neuropsychology and Functional Neuroimaging Research Unit at CRCN, Center for Research in Cognition and Neurosciences, Université Libre de Bruxelles, Brussels, Belgium \\ b LCFC - Laboratoire de Cartographie Fonctionnelle du Cerveau, Université Libre de Bruxelles, Brussels, Belgium \\ ' UNI - ULB Neurosciences Institute, Université Libre de Bruxelles, Brussels, Belgium \\ d Brain Research Unit and MEG Core, O.V. Lounasmaa Laboratory, Aalto University School of Science, Espoo, Finland
}

\section{A R T I C L E I N F O}

\section{Article history:}

Accepted 5 October 2014

Available online 12 October 2014

\section{Keywords:}

Magnetoencephalography

Mu rhythm

Motor learning

Power change

Rebound

Aging

\begin{abstract}
A B S T R A C T
Modulation of the mu-alpha and mu-beta spontaneous rhythms reflects plastic neural changes within the primary sensorimotor cortex (SM1). Using magnetoencephalography (MEG), we investigated how aging modifies experience-induced plasticity after learning a motor sequence, looking at post-vs. pre-learning changes in the modulation of mu rhythms during the execution of simple hand movements. Fifteen young (18-30 years) and fourteen older (65-75 years) right-handed healthy participants performed auditory-cued key presses using all four left fingers simultaneously (Simple Movement task - SMT) during two separate sessions. Following both SMT sessions, they repeatedly practiced a 5-elements sequential finger-tapping task (FTT). Mu power calculated during SMT was averaged across 18 gradiometers covering the right sensorimotor region and compared before vs. after sequence learning in the alpha $(9 / 10 / 11 \mathrm{~Hz})$ and the beta $(18 / 20 / 22 \mathrm{~Hz})$ bands separately. Source power maps in the mu-alpha and mu-beta bands were localized using Dynamic Statistical Parametric Mapping (dSPM). The FTT sequence was performed faster at retest than at the end of the learning session, indicating an offline boost in performance. Analyses conducted on SMT sessions revealed enhanced rebound after learning in the right SM1, 3000-3500 ms after the initiation of movement, in young as compared to older participants. Source reconstruction indicated that mu-beta is located in the precentral gyrus (motor processes) and mu-alpha is located in the postcentral gyrus (somatosensory processes) in both groups. The enhanced post-movement rebound in young subjects potentially reflects post-training plastic changes in SM1. Age-related decreases in post-training modulatory effects suggest reduced experience-dependent plasticity in the aging brain.
\end{abstract}

(c) 2014 Elsevier Inc. All rights reserved.

\section{Introduction}

The ability to learn complex sequences of movements is a necessary requisite for many everyday motor activities. In addition, motor skills acquired during training continue to develop offline after practice has ended, while awake or asleep. Offline processes taking place after the end of the training period participate in memory consolidation, allowing newly acquired abilities to become increasingly stable and resistant to interference through the passage of time (Dudai, 2004; Frankland and Bontempi, 2005; McGaugh, 2000; Walker and Stickgold, 2006). Learning and consolidation of motor skills are accompanied by functional and structural reorganization within specific cerebral regions

\footnotetext{
* Corresponding authors at: UR2NF - Neuropsychology and Functional Neuroimaging Research Unit, Université Libre de Bruxelles (ULB), CP191, Avenue F.D. Roosevelt 50, 1050 Brussels, Belgium.Fax: + 3226502209.

E-mail addresses: alismary@ulb.ac.be (A. Mary), philippe.peigneux@ulb.ac.be (P. Peigneux)
}

(Peigneux et al., 2006), especially in the primary motor cortex (M1) known to be involved in motor learning (Censor et al., 2010; Karni et al., 1995; Muellbacher et al., 2002; Robertson et al., 2005). The functional role of M1 in motor sequence learning can be defined as the storage site for new motor memories, where representations of the sequence are progressively strengthened in specific networks of neurons, supporting practice-related plastic changes in motor maps (Penhune and Steele, 2012). Besides M1, several other brain regions are involved in the acquisition and consolidation of motor sequence learning. Motor skill learning is supported by cortico-cerebellar and cortico-striatal systems (e.g., Doyon et al., 2009; Penhune and Steele, 2012). The cerebellum has been hypothesized to participate to sensorimotor integration and error correction that facilitate the first phases of learning (Penhune and Steele, 2012). Activity in the cerebellum decreases with practice of the motor sequence. By contrast, the striatum and associated motor cortical regions remain activated when the sequence of movement is well learned, supporting their role in long-term consolidation (Doyon et al., 2009). The striatum might play an important 
role for learning associations between individual movements in the sequence (e.g. motor chunks) (Penhune and Steele, 2012).

Young participants trained on a motor sequence exhibit a strong but transient offline improvement of motor performance after an inactivity period of 5-30 min following training (Albouy et al., 2006; Hotermans et al., 2006, 2008; Schmitz et al., 2009), which is not present anymore after $4 \mathrm{~h}$ of inactivity or during uninterrupted practice (Hotermans et al., 2006). The amplitude of the performance boost predicts the size of the performance gains observed $48 \mathrm{~h}$ after learning, suggesting a functional implication of the boost phenomenon in motor memory processes (Hotermans et al., 2006). However, repetitive transcranial magnetic stimulation (rTMS) applied over M1 immediately after the end of learning, and before the motor sequence test, reduces the boost effect but does not impact delayed, overnight gains in performance (Hotermans et al., 2008). Similarly, TMS applied over M1 immediately after practice hinders improvements in motor memory over the day, but not overnight (Robertson et al., 2005). Altogether, these results suggest that M1 plays a critical role during the motor training phase and during the immediately ensuing post-learning wake period, but that different neural networks are already involved in longer-term motor memory consolidation processes (Hotermans et al., 2008; Robertson et al., 2005), indicating that the boost effect reflects a temporary activated state of motor memory (Hotermans et al., 2008). This temporary improvement in motor performance is independent of the practiced material as it generalizes also to untrained sequences (Schmitz et al., 2009), suggesting that the motor boost subtends the optimization of general features of motor learning, rather than consolidating specific sequential components of the material to be learned.

Memory and plasticity processes are crucial players in the maintenance and optimization of cognitive functioning in aging (Lupien and Wan, 2004; Walker, 2005). Several studies evidenced a decline in M1 plasticity with aging, which might be responsible for motor deficits (Freitas et al., 2011; Sawaki et al., 2003; Todd et al., 2010). However, recent studies suggest that older adults are as efficient as young adults at the initial encoding phase of motor learning, and that potential difficulties may appear later on during the offline memory consolidation phase (Brown et al., 2009; Spencer et al., 2007; Wilson et al., 2012). The boost and memory consolidation phases are distinctive in that the former is transiently present after only a few minutes, whereas the latter sustains and takes hours to days to achieve (Press et al., 2005). To the best of our knowledge, the potential impact of aging on the boost of motor performance following a 5-30 min offline resting period has never been investigated. As well, how reorganization processes take place in M1 following learning in young and older participants has not been specifically studied either.

Activity in the primary sensorimotor cortex (SM1) can be assessed by measuring changes in the rolandic mu rhythm with dominant frequencies in alpha $(\sim 10 \mathrm{~Hz})$ and beta $(\sim 20 \mathrm{~Hz})$ frequency bands (Cheyne, 2012; Hari and Salmelin, 1997; Jones et al., 2009). Mu-alpha and mu-beta may involve distinct functional networks (Hari and Salmelin, 1997; Hari, 2006; Ritter et al., 2009). The beta rhythm would originate in the pre-central gyrus, responsible for motor functions, whereas the alpha rhythm would be generated more posteriorly, in the post-central gyrus, responsible for somatosensory processes. Power decrease or increase in the ongoing mu rhythm is known as event-related desynchronization (ERD) or synchronization (ERS), respectively (Pfurtscheller and Lopes da Silva, 1999). The mu rhythm observed at rest is transiently reduced during movement execution, observation, imagination and preparation (Chatrian et al., 1959; Cheyne et al., 2003; Hari, 2006; Hari and Salmelin, 1997; Neuper et al., 2006; Pineda, 2005), and then enhanced after movement termination (Jurkiewicz et al., 2006; Neuper et al., 2006; Pfurtscheller et al., 1996). This post-movement mu rebound overtakes pre-movement levels and lasts a few seconds before returning to baseline (Pfurtscheller et al., 1996). The mu ERD is believed to represent an active state of motor and/or sensory networks during movement preparation and execution
(Jurkiewicz et al., 2006), whereas the mu ERS would reflect active cortical inhibition (Gaetz et al., 2010; Klimesch et al., 2007), cortical stabilization (Caetano et al., 2007) or active immobilization (Erbil and Ungan, 2007; Salmelin et al., 1995) of the SM1. More recently, it has also been suggested that ERS may represent an active process in SM1 that promotes the maintenance of the current movement, i.e. the "status quo" hypothesis (Engel and Fries, 2010). Theta burst stimulation over M1 induces an increased beta power that may represent Long-Term Depression (LTD) plasticity mechanisms (McAllister et al., 2013; Noh, et al., 2012). Importantly, developing expertise in a motor task may also require modulation of the cortical mu rhythm to integrate the new motor ability. Enhanced modulation of the mu rhythm was evidenced during motor skill acquisition (Boonstra et al., 2007), with a stronger decrease in beta power and improved proficiency in bimanual sequence execution (Andres et al., 1999), a greater decrease in alpha power during repetitive sensory stimulation associated with subsequent perceptual learning improvement (Freyer et al., 2013), and a progressive decrease in the $10-\mathrm{Hz}$ power coupled with increased explicit knowledge of the motor sequence (Zhuang et al., 1997). These changes in the modulation of the mu rhythm might reflect plasticity processes taking place in M1 during the course of motor learning. However, to the best of our knowledge, there is nowadays no study evidencing a role of $\mathrm{mu} \mathrm{ERD} / \mathrm{S}$ in the motor plastic changes that take place after a specific and complex motor learning.

In the present study, we hypothesized that modulation of mu rhythms over M1 during simple movements would be influenced by a prior, complex motor sequence learning experience, in line with studies suggesting an involvement of M1 within the first minutes (Hotermans et al., 2008) or hours (Robertson et al., 2005) after learning. Supporting this hypothesis, previously published MEG investigations disclosed increased beta modulation in M1 (i.e. increased beta ERD and ERS) during motor learning positively correlated with motor performance, suggesting that increased modulation may reflect M1 functional reorganization during the time course of motor learning (Boonstra et al., 2007). In line with these findings, increased modulation of the mu rhythm following motor sequence learning during the execution of simple movements may reflect a practice-dependent sensorimotor reorganization. However, mere movement repetition may result in a progressive ERD decrease, more directly related to an habituation effect reducing attentional demands directed towards the motor task (Dirnberger et al., 2004; Erbil and Ungan, 2007). Experience-dependent modulation of the cortical mu rhythm following learning might be diminished with aging, in line with studies suggesting an age-related decline in M1 plasticity following motor learning (Freitas et al., 2011; Sawaki et al., 2003; Todd et al., 2010). To test these predictions, we investigated the modulation of mu rhythms using magnetoencephalography (MEG) during the execution of simple hand movements (Simple Movement Task, SMT) after learning a motor sequence of finger movements (Finger Tapping task, FTT; Karni et al., 1995).

\section{Materials and methods}

\section{Participants}

Fifteen young ( 7 females; range $18-30$ years; $24.26 \pm 3.33$ ) and fourteen older ( 8 females; range $65-75$ years; $69.1 \pm 1.46$ ) right-handed healthy participants gave their written agreement to participate in this MEG study approved by the ULB-Hospital Erasme Ethics Committee. Participants had no history of neuropsychiatric disease or movement disorders and were right-handed according to the Edinburgh handedness inventory (Oldfield, 1971). Participants were screened for depression using the Shortened version of the Beck Depression Inventory (BDI, Beck et al., 1974; French adaptation by Collet and Cottraux, 1986). Sleep habits were assessed using the Pittsburgh Sleep Quality Index (PSQI, Buysse et al., 1989) and the scores were similar in both groups $(t(27)=0.86 ; p=0.4$; young: $4.2 \pm 2.98$; older: $3.36 \pm 2.24)$. 
Participants did not take any medication that could have affected sleep or memory. All had neutral or intermediate chronotype, as measured by the Morningness-Eveningness Questionnaire (Horne and Ostberg, 1976). Older (14.86 \pm 3.21$)$ and young participants $(15.8 \pm 3.49)$ did not significantly differ in years of education $(t(27)=0.75, p=0.46)$. Additionally, older participants were screened for dementia using the Mattis Dementia Rating Scale (Mattis, 1976); all scored within the normal range (mean $=141.42 / 144 \pm 1.89$ ). Before data acquisition, participants were administered the State-Trait Anxiety Inventory (STAI, French version: Bruchon-Schweitzer and Paulhan, 1990) to determine both state- and trait-levels of anxiety, and the St Mary's Hospital sleep questionnaire (adapted from Ellis et al., 1981) to evaluate the quality of sleep on the night preceding the experiment.

\section{Experimental procedure}

The experimental design is illustrated Fig. 1. The experiment was programmed and conducted using Cogent 2000 and Cogent Graphics (http://www.vislab.ucl.ac.uk) software implemented in MATLAB 6.1 (Mathworks Inc., Sherbom, MA). Stimuli were back-projected on a screen $(32 \times 51 \mathrm{~cm})$ placed $1 \mathrm{~m}$ in front of the participants' eyes. Information was written in light gray, font Arial size 25 on a black background, with a $1024 \times 768$ pixels screen resolution.

\section{Simple movement task (SMT)}

In the SMT, subjects pressed the four keys using the four fingers simultaneously every $5 \mathrm{~s}$, in response to an auditory cue. The task comprised 100 trials, and subjects had to look at a centrally located fixation cross at all time to avoid eye movement artifacts. The task was performed during two separate MEG sessions, before and after the learning FTT phase (see below).

\section{Finger tapping task (FTT)}

In the FTT, subjects had to reproduce as fast and accurately as possible a five-element sequence of 4 possible key presses using the four fingers of the left, non-dominant hand (sequence [4-1-3-2-4] from index [1] to little finger [4]). The non-dominant hand was used to avoid ceiling effect with the dominant hand as motor dexterity (i.e. precision and speed) is decreased for the non-dominant hand as compared to the dominant hand (Francis and Spirduso, 2000). Each trial consisted of 3 elements (Orban et al., 2011): preparation, sequence reproduction and a 10 -second rest. During the preparation phase, subjects mentally planed the sequence. During the execution phase, subjects started reproducing the sequence whenever they felt ready; the sequence had to be repeated twice. An auditory cue was then delivered following the last element of the sequence and participants had to relax during $10 \mathrm{~s}$, allowing the mu rhythm to be back to baseline amplitude level (Cassim et al., 2000). During the preparation and execution phases, a numeric representation of the sequence to perform was displayed on the screen [4-1-3-2-4], and replaced by a [X-X-X-X-X] pattern during the resting period. Subjects performed the FTT task immediately after the first SMT session (learning phase; 70 trials) and after the second SMT session (retest phase; 50 trials).
A Visual Analogue Scale of Fatigue (VASF) was completed before and after the MEG session in order to identify fatigue effects and potential differences between groups. In addition, participants had to score their ability to prepare the motor execution of the sequence, ranging from 1 (very hard to feel) to 7 (very easy to feel) on a Likert kinesthetic imagery scale (adapted from Hall and Martin 1997). Subjective scores were similar between groups (young $5.03 \pm 1.46$ vs. older $5.34 \pm 1.19 ; \mathrm{t}(27)=-0.62$; $\mathrm{p}=0.54$ ) and participants considered on average the sequence as "quite easy to feel".

\section{Finger tapping task measures and statistical analyses}

Behavioral performance on the FTT was computed considering the 8 possible three-element chunks that composed each trial (i.e. 413, 132, $324,244,441,413,132,324$ ), as previously published (Hotermans et al., 2006, 2008). With practice indeed, the motor sequence is not conceived anymore as constituted of individual successive elements, but rather becomes a temporal representation associating elements together and reorganizing the sequence in a motor chunk (Bo and Seidler, 2009; Cleeremans and McClelland, 1991; Verwey, 2010). Chunk length was limited to three elements given evidence that young subjects are able to encode 3 to 4 temporal elements together (Bo and Seidler, 2009), and that elders are able to use a maximum of 4 elements during motor sequence learning (Bo et al., 2009). Speed performance was computed as the time needed to correctly generate the 3-element chunks from the 2 sequences executed per trial (execution times). As well, accuracy score was computed as the percentage of correctly generated 3-element chunks (percentage of correct responses). Comparisons within and between groups were computed using repeated-measures ANOVA conducted on mean execution times and percentage of correct responses. Within-session effects were assessed comparing the average of the first and last 20 trials of each session (learning and retest). The boost effect was calculated comparing execution time for the 20 last trials of the learning session (trials 51-70) to execution time for the 20 first trials of the retest session (trials 3-22). The two first trials of both sessions were removed from statistical analysis to allow habituation to the task.

\section{Additional finger tapping task measures (behavioral-only session)}

The boost effect is a transient motor phenomenon (Albouy et al., 2006; Hotermans et al., 2006, 2008; Schmitz et al., 2009), that might be dampened by the repetition of movements in the SMT interleaved between the FTT learning and retest phases, as well as by the constraints of the experimental design in the MEG environment (e.g. cued production of the FTT sequence, see discussion below for further details). Consequently, we conducted a supplementary experiment with 14 young (10 females; range $18-30$ years; $19.71 \pm 1.54$ ) and 12 older ( 7 females; range $65-75$ years; $69.83 \pm 3.26$ ) right-handed healthy subjects, using our previously published setting (Hotermans et al., 2006, 2008). Participants practiced the same 5-element sequential FTT during 12 blocks of $30 \mathrm{~s}$ each, interleaved with 30 -second rest periods. Performance was tested 30 min after learning on 2 additional blocks. The 30-min interval between learning and retest was filled with questionnaires. Speed performance was computed as the number of correctly generated 3-element chunks in each block (Hotermans et al., 2006, 2008). The first learning block was removed from statistical analysis to allow

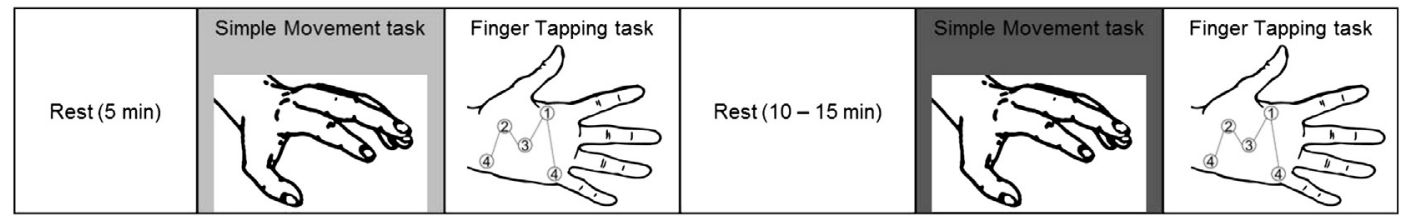

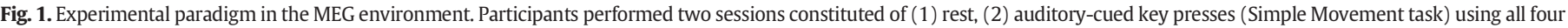

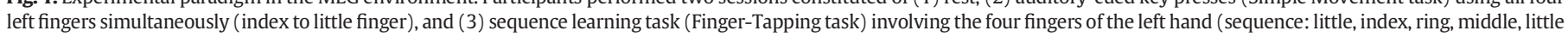
fingers). MEG analyses were realized on Simple Movement task (SMT) executed before (light gray) and after (dark gray) the sequence learning task. 
habituation to the task and reach optimal accuracy. The learning effect was assessed comparing the number of 3-element chunks generated during the 2 first blocks (block 2-3) and the 2 last blocks (block 11-12) of the learning session. The boost effect was assessed comparing the number of 3-element chunks generated in the 2 last blocks of the learning session (block 11-12) and in the 2 blocks of the retest session (block 13-14). Participants had no history of neuropsychiatric disease or movement disorders and were right-handed according to the Edinburgh handedness inventory (Oldfield, 1971). No musicians or professional typists were included. Depression scores on the Shortened version of the Beck Depression Inventory (BDI, Beck et al., 1974; French adaptation by Collet and Cottraux, 1986) for the young participants and on the Geriatric Depression Scale (GDS-15, Sheikh and Yesavage, 1986) for older participants were below cut-off scores in both groups. Sleep habits were assessed using the Pittsburgh Sleep Quality Index (PSQI, Buysse et al., 1989) and were also similar $(t(27)=0.86 ; p=0.4$; young: $4.2 \pm 2.98$; older: $3.36 \pm 2.24)$. Participants did not take any medication that could have affected sleep or memory. Older adults averaged an intermediate morning-type $(62.79 \pm 6.44)$ whereas young adults averaged a neutral type $(43.64 \pm 6.81 ; t(24)=-7.33$, $p<0.001$ ) in the Morningness-Eveningness Questionnaire (Horne and Ostberg, 1976). Older participants were screened for dementia and scored within the normal range (score $\geq 26,27.92 \pm 1.08$ ) at the Montreal Cognitive Assessment (MoCa; Nasreddine et al., 2005).

\section{MEG data acquisition}

MEG signals were recorded in a light-weight magnetically shielded room using a whole-scalp 306-channel system (Vectorview \& Maxshield ${ }^{\mathrm{TM}}$; Elekta Oy, Helsinki, Finland). Head position inside the MEG helmet was continuously monitored using four head tracking coils. The locations of the coils and at least 150 head-surface (on scalp, nose and face) points were determined with respect to three anatomical landmarks (nasion and preauricular points) using an electromagnetic digitizer (Fastrak, Polhemus, Colchester, VT, USA). Electrooculogram (EOG) signals were obtained using bipolar electrodes located below the left outer canthus and above the right outer canthus. Electrocardiogram (ECG) was recorded with bipolar electrodes placed below the right collarbone and at the left last rib. Movements of the left hand were monitored using bipolar electrodes placed on the left extensor carpi ulnaris muscle. Individual 3D-T1 weighted Magnetic Resonance Imaging (MRI) was also acquired using a 1.5 T MRI scanner (Intera, Philips, The Netherlands) at ULB-Hôpital Erasme.

\section{MEG data pre-processing}

Continuous MEG data were pre-processed using the signal space separation (SSS) method to suppress external interferences and correct for head movements (Taulu et al., 2005), and band-pass filtered in the $1-45 \mathrm{~Hz}$ frequency range. Epochs were extracted from $-1000 \mathrm{~ms}$ to $4000 \mathrm{~ms}$ relative to auditory stimuli onsets during SMT. Epochs containing artifacts (e.g. muscle artifacts) were removed from further analyses based on visual inspection. An Independent Component Analysis was performed for each subject to identify and remove components reflecting cardiac and ocular activity. Ocular and cardiac components were selected on the basis of their topography and correlations with EOG and ECG signals, respectively. MEG epochs exceeding 3 pT (magnetometers) or $0.7 \mathrm{pT} / \mathrm{cm}$ (gradiometers) were further excluded from the analysis to discard any remaining artifact in the MEG signals.

\section{MEG data analyses}

Time-frequency analysis at the sensor level. Time-frequency decomposition of all epochs was obtained with wavelet transformation, using a 7-cycle Morlet wavelet (Tallon-Baudry et al., 1996). Power was averaged over a pre-selection of 18 gradiometers overlying the right and left sensorimotor region (Kim and Chung, 2007). For each subject, the time course of alpha and beta oscillations was assessed at the frequency yielding the maximal power (alpha: 9/10/11 Hz; beta: 18/20/22 Hz). The period used for statistical analysis started $1000 \mathrm{~ms}$ prior to the auditory cue triggering the simple movements and lasted $4000 \mathrm{~ms}$. The time course of the power increase/decrease in the alpha and beta bands relative to the baseline value (from -800 to $-200 \mathrm{~ms}$ ) were obtained as follows: $\left(\mathrm{P}(\mathrm{t}, \mathrm{f})-\mathrm{P}_{\text {baseline }}(\mathrm{f})\right) / \mathrm{P}_{\text {baseline }}(\mathrm{f})$.

Statistical differences in power within each group and for each frequency band (alpha and beta) were conducted for the SMT before vs. after the FTT learning session and were computed using Wilcoxon signed rank test performed on the power averaged in 500-ms adjacent windows spanning the 0 to $4000-\mathrm{ms}$ range. Between-group comparisons in changes after vs. before FTT learning were conducted in the alpha and beta bands separately using permutation tests (Nichols and Holmes, 2002). First, power differences after vs. before FTT learning were calculated for each participant, then power differences were averaged within the young and older groups. Second, power differences after vs. before FTT learning in the older group were subtracted from power differences in the young subjects. Two variables were calculated by period of $500 \mathrm{~ms}$ duration (spanning the 0 to 4000 -ms range): the maximum absolute power differences, and the average absolute power differences. Third, the sampling distribution of these variables was assessed computing their values after randomly shuffling young and older participants (1000 permutations). Results were deemed significant when they exceeded the 95-percentile of their sampling distribution.

Finally, associations between sequence learning performance and power changes after vs. before FTT learning were assessed using a linear regression analysis with differences in power change between young and older participants as dependent variable and with the withinsession gain during learning, the between-session gain (30-min boost) and the age of our participants as predictors. The within-session gain during learning was computed as the percentage of improvement from the 20 first trials to the 20 last trials of learning and the between-session gain as the percentage of improvement from the 20 last trials of learning to the 20 first trials of retest.

Source reconstruction. Individual MRIs were segmented using Freesurfer software (Martinos Center for Biomedical Imaging, Massachusetts, USA). Co-registration between MEG and MRI coordinate systems was achieved using the three anatomical landmarks for initial estimation and the head-surface points to manually refine the surface co-registration. Individual MEG forward models were then computed using the Boundary Element Method implemented in the MNE software suite (Martinos Center for Biomedical Imaging, Massachusetts, USA). To perform group averaging, the MEG forward models were first defined on a source grid obtained from a homogeneous 5-mm grid source space covering the whole Montreal Neurological Institute (MNI) brain, and then transformed onto individual MRIs using the non-linear spatialnormalization algorithm implemented in Statistical Parametric Mapping (SPM8, Wellcome Department of Cognitive Neurology, London, UK). Individual sources power maps in the mu-alpha and mu-beta bands were then reconstructed using Dynamic Statistical Parametric Mapping (dSPM) (Dale and Sereno, 1993) on the basis of the mu rebound estimated during SMT executed before learning. We first used sensor-level timefrequency analysis (see Time-frequency analysis at the sensor level) to identify at the individual level post-movement time and frequency (alpha: 9/10/11 Hz; beta: $18 / 20 / 22 \mathrm{~Hz}$ ) of maximum power increase with respect to the baseline ( -800 to $-200 \mathrm{~ms}$ pre-movement) in right sensorimotor sensors. The associated sensor-level wavelet coefficients where source-projected with dSPM (noise covariance matrix estimated from pre-movement baseline $(-800$ to $-200 \mathrm{~ms})$ filtered at the same frequency) and the ensuing relative power increases were then computed. The resulting individual mu-alpha and mu-beta power maps were finally averaged separately over the young and older subjects to create group-level maps, which were displayed on the MNI brain in order to localize the mu-alpha and mu-beta rhythms. 
Table 1

Scores on VASF, STAI, PSQI, and St-Mary questionnaires (means and standard deviations).

\begin{tabular}{lllll}
\hline \multicolumn{4}{l}{ Young adults } & \multicolumn{2}{l}{ Older adults } \\
\hline VASF & & & & \\
Drowsiness before (\%) & 28.6 & $(22.05)$ & 23.08 & $(15.9)$ \\
Drowsiness after (\%) & 38.5 & $(25.44)$ & 37.23 & $(17.26)$ \\
Fatigue before (\%) & 41.24 & $(26.2)$ & 20.62 & $(15.51)$ \\
Fatigue after (\%) & 40.14 & $(27.16)$ & 32.38 & $(17.16)$ \\
STAI & & & & \\
State & 30.33 & $(9.98)$ & 26.23 & $(5.23)$ \\
Trait & 37 & $(9.14)$ & 33 & $(5.51)$ \\
PSQI & 7.44 & $(1.14)$ & 7.39 & $(1.05)$ \\
Sleep duration (hours) & 25 & $(28.16)$ & 12.46 & $(16.28)$ \\
Sleep latency (minutes) & & & \\
St Mary's Hospital sleep questionnaire & & 7.13 & $(1.14)$ \\
Sleep duration (hours) & 7.34 & $(1.12)$ & 12.38 & $(12.27)$ \\
Sleep latency (minutes) & 14.67 & $(14.33)$ & & \\
\hline
\end{tabular}

Note: Percentage of drowsiness and fatigue were assessed twice for the VASF, i.e. before and after MEG acquisition.

\section{Results}

Questionnaires (see Table 1)

Right-handedness measured by the Edinburgh handedness inventory (Oldfield, 1971) was less pronounced in young $(75.33 \% \pm 16.31)$ than older $(87.86 \% \pm 12.97)$ participants $(t(27)=-2.28, p=0.03)$. Health condition scored on a Likert-type rating scale, ranging from 0 (very bad) to 10 (excellent), was similar between groups $(t(27)=1.47$; $p=0.15$; young: $8.37 \pm 1.008$; older: $7.86 \pm 0.84$ ). Similarly, depression scores on the Shortened version of the Beck Depression Inventory (BDI, Beck et al., 1974; French adaptation by Collet and Cottraux, 1986) were similar and below cut-off score $(t(27)=0.42$; $p=0.68$; young: $2.67 \pm 2.53$; older: $2.29 \pm 2.33$ ). Chronotype was measured by the Morningness-Eveningness Questionnaire (Horne and Ostberg, 1976). Older adults averaged an intermediate morning-type $(60.75 \pm 7.33)$ whereas young adults averaged a neutral type $(49.71 \pm 7.34 ; t(27)=-3.97, p<0.001)$.

A repeated measures ANOVA performed on drowsiness and fatigue VASF scores with within-factor time of assessment (before vs. after MEG acquisition) and between-factor group revealed a main effect of time of assessment for drowsiness $(F(1,26)=11.53 ; p<0.01)$, indicating that subjects were more drowsy at the end than at the beginning of the testing session. Other effects and interactions were not significant. STAI (Bruchon-Schweitzer and Paulhan, 1990) questionnaire values were similar between groups ( $p s>0.1$ ). Sleep duration and sleep latency were evaluated with both the PSQI (Buysse et al., 1989) to estimate participants' sleep habits during the last month, and the St Mary's Hospital sleep questionnaire (Ellis et al., 1981) to estimate the maintenance of these habits during the night preceding the MEG acquisition. Repeated measures ANOVA performed on sleep duration and sleep latency showed that these sleep variables did not differ between questionnaires ( $p s>0.1$ ), indicating that participants kept their sleep habits during the night preceding the MEG acquisition. The main effect of group and the interaction effects were non-significant ( $p s>0.1$ ), suggesting that sleep latency and duration were similar between groups.

\section{Finger tapping task}

Execution times (speed performance) are illustrated Fig. 2 for both groups at training and retest MEG sessions.

A repeated measures ANOVA for the 3-element chunks (Fig. 2B) with session (learning vs. retest) and trial type (20 first vs. 20 last) as within-subject factors and group (young vs. older) as betweensubject factor yielded significant effects of session $(F(1,27)=18.86$; $p<0.001)$ and trial type $(F(1,27)=34.01 ; p<0.001)$, and a session by trial interaction effect $(F(1,27)=30.13 ; p<0.001)$. The group effect was non-significant $(F(1,27)=2.05 ; p=0.16$, $)$, as well as other interactions $(p>0.15)$. Tukey's post-hoc tests disclosed faster execution times in the last than the first trials of the learning session (1032 \pm $363 \mathrm{~ms}$ vs. $854 \pm 258 \mathrm{~ms}, p<0.001$ ) and faster execution times in the beginning of the retest session as compared to the end of the learning session $(854 \pm 258 \mathrm{~ms}$ vs. $779 \pm 210 \mathrm{~ms}, p=0.01)$, indicating a boost effect on performance. No significant difference in execution times was identified between the beginning and the end of the retest session $(p<0.82)$. The same statistical analyses were computed for the full sequence of five elements (Fig. 2A). Results for the full sequence of five elements are similar to the results with the 3-element chunks.

A repeated measures ANOVA conducted on the percentage of correct responses revealed a significant session by trial interaction $(F(1,27)=5.46, p<0.05)$, but Tukey's post-hoc tests did not evidence any significant differences ( $p s>0.1$ ).
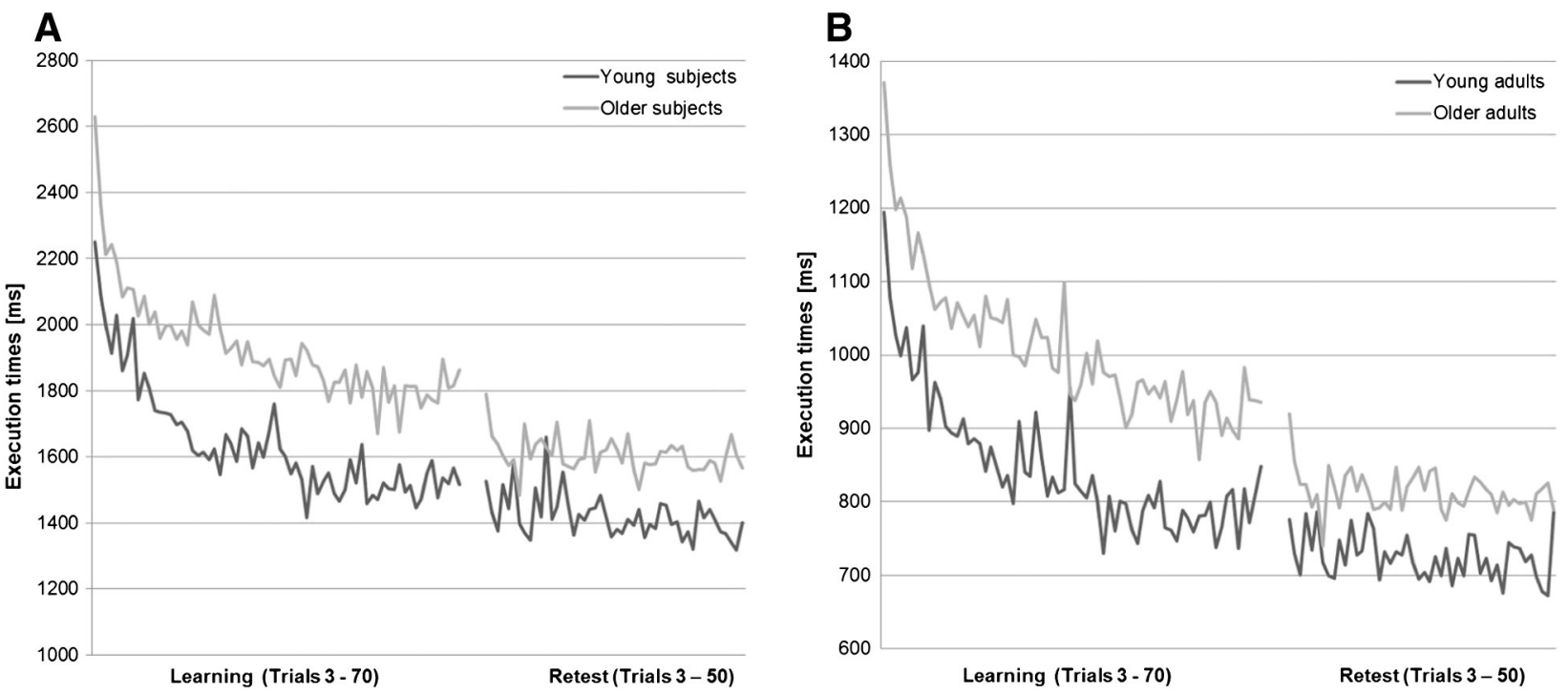


the training and retest sessions. B. Mean execution times [ms] for 3-element chunks generated per trial during the training and retest sessions. 


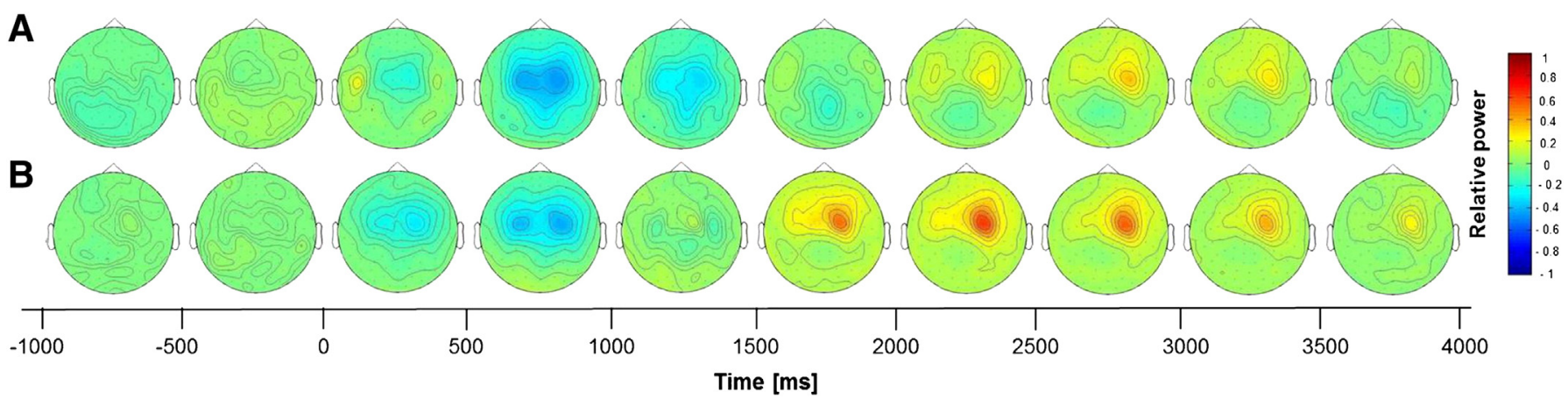

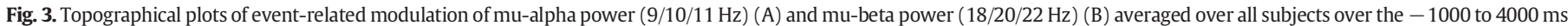
time window during SMT executed after learning. Time 0 on the $\mathrm{x}$ axis is aligned with the onset of the auditory cue triggering simple movements' execution.

\section{MEG measurements}

Time-frequency analysis at the sensor level

Time-frequency analyses were performed to identify age-related differences in the time course of oscillatory changes associated with simple movements (SMT) executed before vs. after learning. Mean latencies before the execution of simple movements following the auditory cue $(528.63 \pm 219.06 \mathrm{~ms})$ and the mean duration of movements $(155.87 \pm 144.12 \mathrm{~ms})$ were similar in both Simple Movement Task (SMT) sessions ( $p s>0.5$ ), suggesting that the way movements were executed remained similar. Therefore, significant neurophysiological differences between SMT sessions (before vs. after the FTT learning) cannot simply be explained by changes in movement latency or duration. The most prominent power changes are observed within the nine pairs of planar gradiometer channels selected for the MEG analyses (Fig. 3).

Within-group comparisons before vs. after the FTT learning showed that the decrease in beta power during movement execution was stronger before than after learning in the left SM1 in older participants (Wilcoxon signed-rank test: $p=0.01$ ). In young participants, the subsequent post-movement rebound was higher after than before motor sequence learning in both alpha and beta bands in the right SM1 (Wilcoxon signed-rank test: $p=0.01$; Fig. 4 ) and in the beta band in the left SM1 (Wilcoxon signed-rank test: $p<0.01$ ).
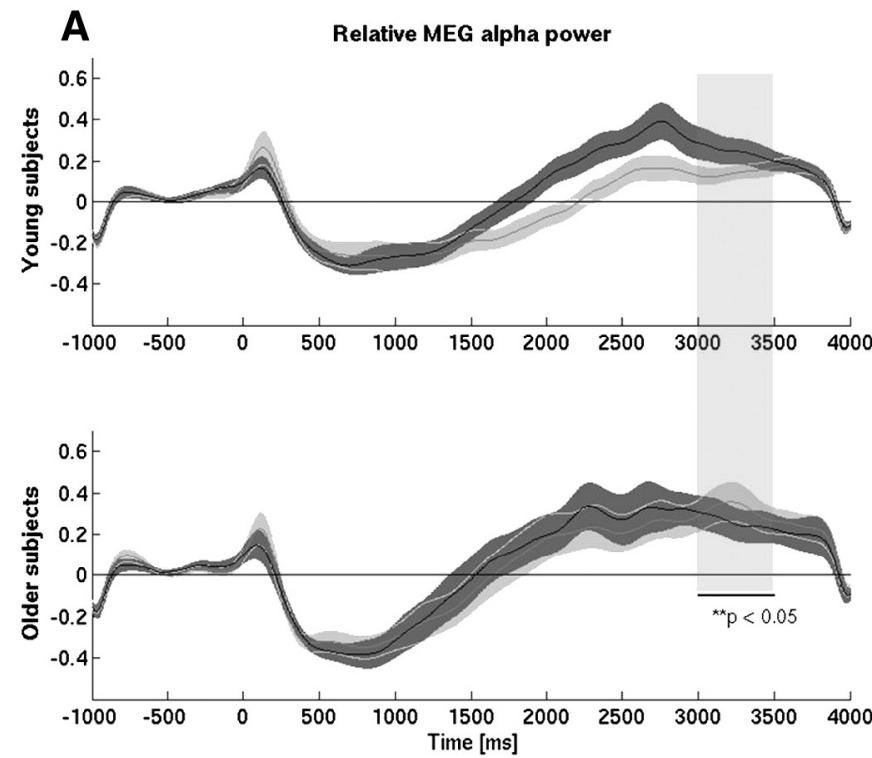

A permutation test disclosed age-related differences in the rebound of the alpha power in the 3000-3500-ms time window, both for the maximum absolute power differences and for the average absolute power differences ( $p s<0.05$; Fig. 4 ). As shown in Fig. 4 , the rebound was enhanced after learning in young but not in older participants in the mu-alpha band in the right SM1. At variance, the rebound after learning in the mu-beta band was not significantly increased in young compared to older participants ( $p s>0.18$ ). All other between-group comparisons were also non-significant ( $p s>0.1$ ).

Finally, in order to determine whether age-related differences in the alpha power rebound are a specific marker of a differential learning effect in aging, we conducted a linear regression analysis with as dependent variable the change in alpha power rebound in the right SM1 and as predictors the within-session gain during learning, the betweensession gain (30-min boost) and the participants' age. The change in alpha power rebound was computed as the difference between the first and second SMT in maximal alpha power obtained from the 1500-4000 ms time window. Two participants ( 1 young, 1 older adult) were excluded from the analysis due to changes in alpha power rebound at more than 2 standard deviations above their group mean. Linear regression analysis revealed that age predicted best changes in alpha power rebound after learning $(t(27)=-2.32 ; p=0.03)$, which was not the case for the within session $(t(27)=-0.55 ; p=0.59)$ or the between session $(t(27)=0.75 ; p=0.46)$ gains.
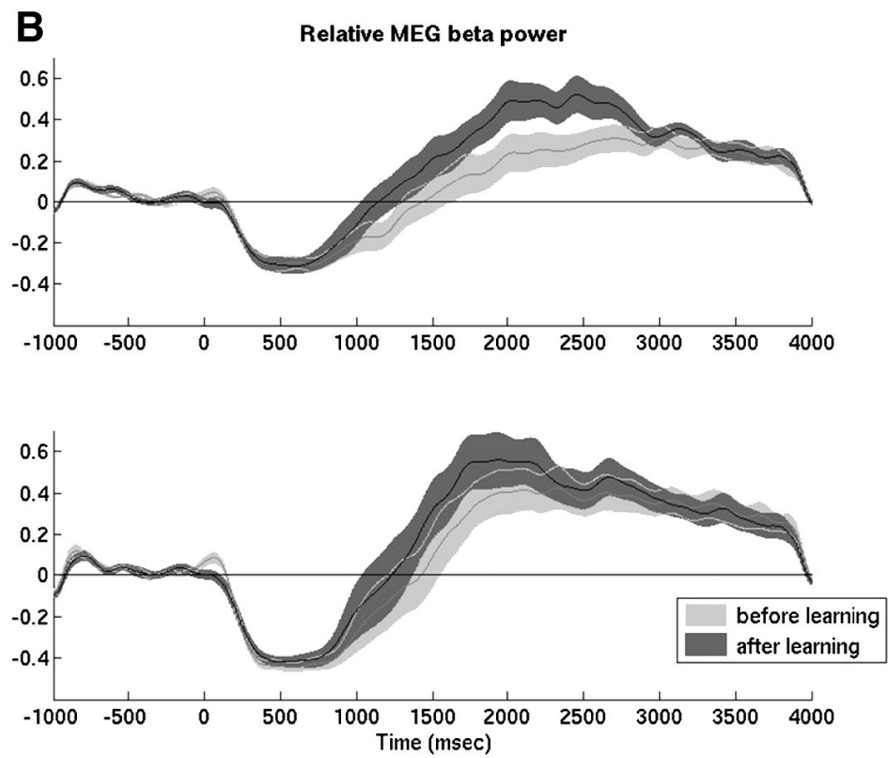

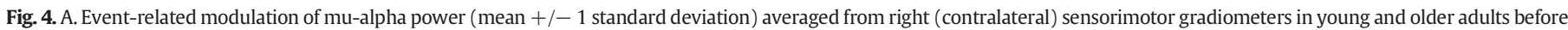

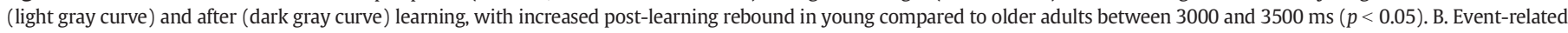

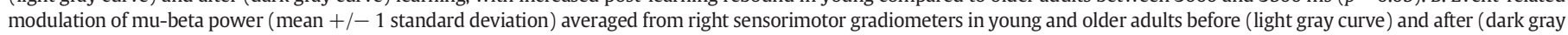
curve) learning. 

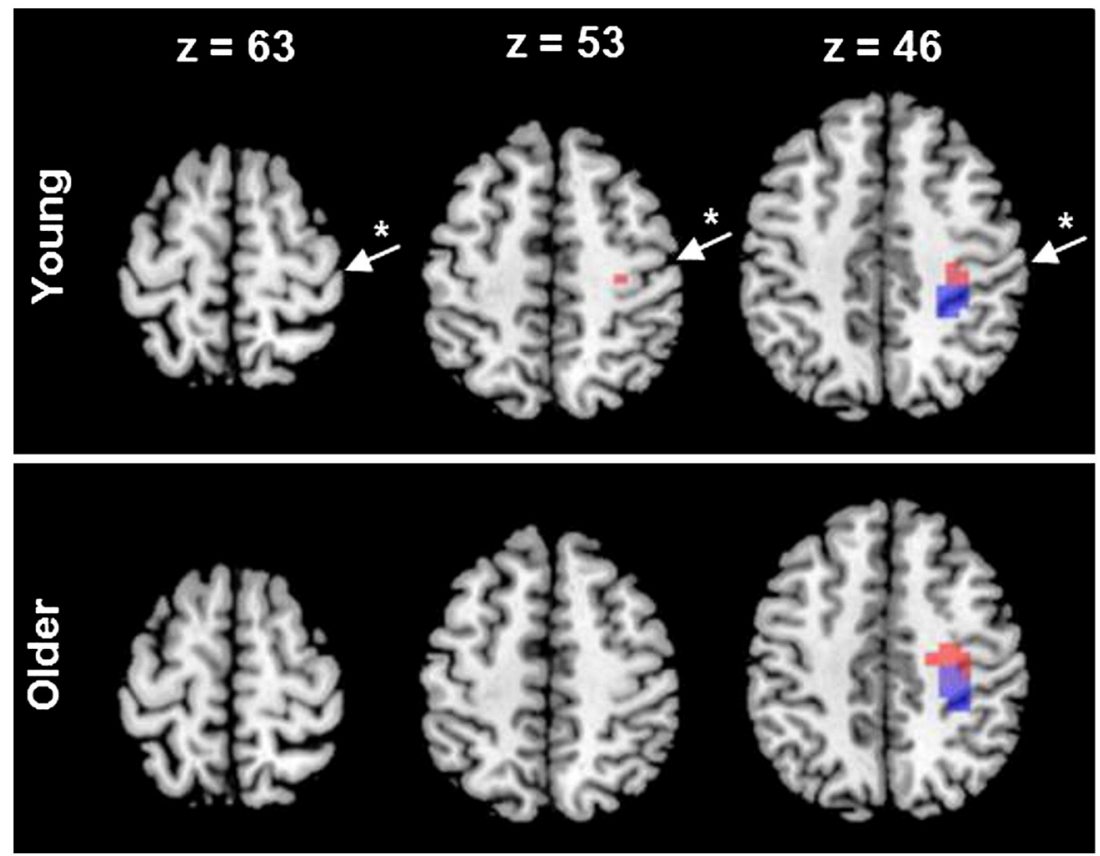

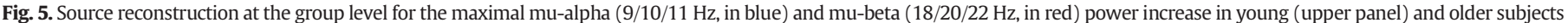
(lower panel). The figure illustrates the same 3 axial slices $(z=63,53$ and $46 \mathrm{~mm}$ in MNI space) for the young and older groups. The white arrows* represent the central sulcus.

\section{Source reconstruction}

Fig. 5 shows the sources of the mu-beta and the mu-alpha rebound in young and older adults separately. Mu-beta is located in the precentral gyrus (anterior to the central sulcus) and mu-alpha is located in the postcentral gyrus (posterior to the central sulcus). Similar localizations are evidenced in young and older subjects for both frequency bands.

\section{Additional FTT (behavioral-only session)}

A repeated measures ANOVA conducted on the number of correctly generated chunks during learning (Fig. 6) revealed a group effect $(F(1,24)=5.93, p<0.05)$ and a learning effect $(F(1,24)=54.48$, $p<0.001)$ but no group by learning interaction $(F(1,24)=0.77$, $p=0.39$ ) effect. The group effect indicated that older adults generated

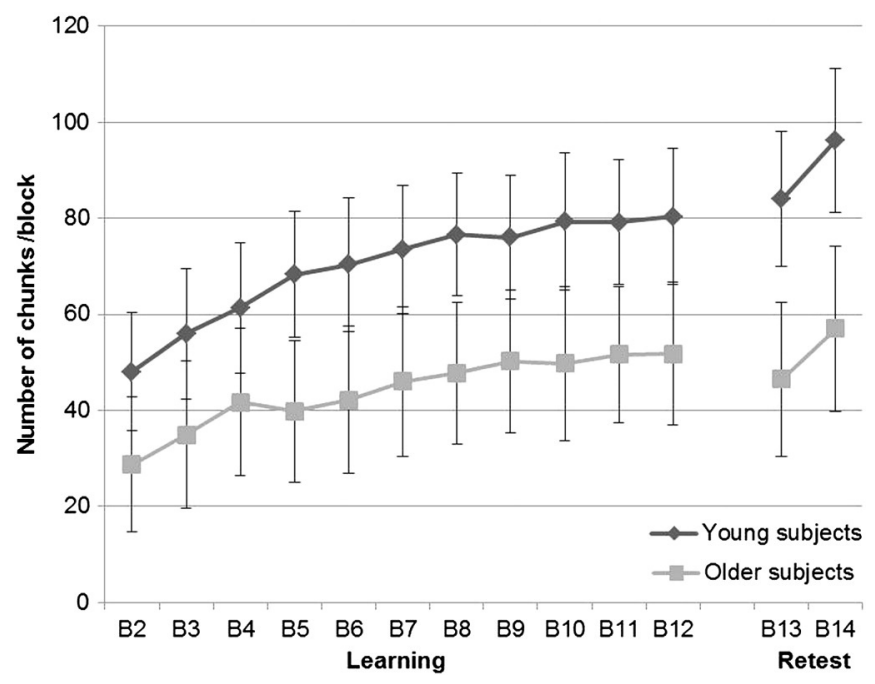

Fig. 6. Finger Tapping task performance during a behavioral-only session in additional young and older samples. Values are number of correctly generated 3-element chunks per 30 -second block during training and retest after $30 \mathrm{~min}$. fewer chunks than young adults within the 30 -second execution time at each trial, which indicates that they were globally slower to generate the sequence. Between session effects, aimed at investigating the boost of performance after $30 \mathrm{~min}$, disclosed a group $(F(1,24)=10.34$, $p<0.01)$ and a group by session interaction $(F(1,24)=5.46$, $p<0.05)$ effects, but no effect of session $(F(1,24)=2.11, p=0.16)$. Tukey's post-hoc tests revealed that performance improved at the 30 min retest as compared to the end of learning in young adults $(p<0.05)$, but remained stable in older participants $(p>0.7)$, suggesting an age-dependent attenuation of the boost phenomenon in the FTT.

\section{Discussion}

The present MEG study aimed at investigating how motor sequence learning modulates plasticity markers, i.e. sensorimotor mu rhythms in the alpha and beta frequency bands during the execution of simple hand movements, and how this experience-dependent modulation is modified with aging.

At the behavioral level in the context of the MEG protocol, the Finger Tapping Task (FTT) was performed faster at retest than at the end of the learning session, indicating an offline boost in performance when considering all participants. However, no differences in performance were observed between young and older adults for this effect. The Simple Movement task (SMT) taking place immediately before the FTT retest did not cancel the boost effect considering all participants but might have attenuated the differences between young and older adults in the boost effect. In our complementary behavioral experiment (in which no intermediate motor task was performed between the learning and retest FTT phases), we replicated the previously observed transient boost of performance (Albouy et al., 2006; Hotermans et al., 2006, 2008; Schmitz et al., 2009) in young but not in older participants. Therefore, a likely explanation for a lack of age-related behavioral differences in the boost effect in the context of the MEG protocol is that the transient and non-specific motor enhancement featuring the boost effect has taken place already during the SMT performed immediately before the FTT retest, at which time it was already exhausted, hence reducing the size of any potential motor skill improvement. Supporting this hypothesis, Hotermans et al. (2008) showed that TMS applied 
over M1 immediately after learning and before retesting the FTT motor sequence masked the behavioral expression of the boost effect. Considering that transient post-learning motor improvement (i.e. a boost effect) reflects the initiation of functional reorganization processes in M1, the post-FTT learning SMT session took place within the 5-30 min during which the boost effect is expressed (Albouy et al., 2006; Hotermans et al., 2006, 2008; Schmitz et al., 2009). Excitability levels in M1 might have been exhausted by the simple hand movements' repetition during the SMT, eventually preventing the differential expression of the boost of performance in younger and older adults. The latter effect was found when not using the SMT task. Accordingly, when participants did not have to perform simple movements immediately before FTT retest, young adults improved performance after a 30minute break whereas older adults merely stabilized their performance. Noticeably, another difference between our MEG and behavioral-only protocols is that in the latter case, participants had the opportunity to produce as much sequences as possible during twelve 30 -second blocks. In the MEG protocol, they had to reproduce as fast as possible the sequence twice every $10 \mathrm{~s}$. Nonetheless, our results show that even without these constraints during learning, older participants cannot improve performance at retest, like their young counterparts. Altogether, our behavioral data suggest reduced motor plasticity with aging, in line with our MEG finding of an attenuated rebound of the mu rhythms.

A progressive attenuation of alpha desynchronization during the course of continuous ( $230 \mathrm{~s}$ ) hand movements might result from habituation processes in the somatosensory cortex (Erbil and Ungan, 2007). Likewise, the amplitude of the movement-related cortical potentials is attenuated during the course of simple repetitive button presses (Dirnberger et al., 2004). In the present MEG study, we found that beta desynchronization in the left SM1 is attenuated after learning in older participants. Such decrease in the ERD amplitude might be related to movement repetition more than to plastic changes associated with previous motor learning. In young participants however, the postmovement rebound was enhanced after motor sequence learning, in both the alpha and beta bands in the right SM1 and in the beta band in the left SM1. This enhancement is in line with a previous report evidencing enhanced event-related beta modulation associated with motor learning (Boonstra et al., 2007). However, our findings did not evidence a stronger decrease in the mu-alpha or the mu-beta power as showed in previous studies on motor learning (Andres et al., 1999; Freyer et al., 2013; Zhuang et al., 1997). Mu rebound is suggested to reflect an active immobilization or inhibition in SM1 (e.g. Klimesch et al., 2007; Salmelin et al., 1995). It was shown previously that inhibitory rebound is proportional to prior activation levels in M1 (Schuch et al., 2010). Since M1 is specifically activated during the first minutes (Hotermans et al., 2008) or hours (Robertson et al., 2005) following motor learning, the immediately preceding sequence learning experiences during the FTT might be responsible for a stronger rebound of the sensorimotor mu rhythms in young adults. In this respect, increased inhibitory control might facilitate practice-dependent plasticity in M1. Indeed, decreased inhibition is surmised to be related to a decline in Long-Term Depression (LTD) plasticity (Todd et al., 2010). Additional results indicate that motor skill learning prevents the subsequent induction of Long-term Potentiation-like (LTP) plasticity but enhances LTDlike plasticity (Ziemann et al., 2004). LTP and LTD are two major mechanisms of learning-induced cortical plasticity. Indeed, LTP increases synaptic connections during learning, facilitating memory storage. Conversely, LTD serves as a homeostatic mechanism dampening memory traces by weakening synaptic connections to avoid synaptic saturation following learning (Cooke and Bliss, 2006; Malleret et al., 2010). An alternative explanation comes from the "status quo" hypothesis (Engel and Fries, 2010) and from the hypothesis supporting that post-movement rebound refers to cortical stabilization in M1 (Caetano et al., 2007). In this perspective, the need of higher mu rebound following learning in young adults would suggest that post-movement cortical stabilization in M1 (Caetano et al., 2007) and/or the maintenance of the simple movement (Engel and Fries, 2010) is more difficult after repetitive sequence learning. This explanatory scheme would support the hypothesis that M1 reorganization is responsible for the following offline motor boost of performance (Hotermans et al., 2008). Additionally, post-learning modulation of mu rhythms during the execution of simple hand movements is in favor of the proposal that the offline motor boost effect is non-specific, therefore independent of the learned sequence (Schmitz et al., 2009).

Our regression analyses evidenced age-related post-motor learning changes in the mu-alpha power rebound, although no behavioral differences could be evidenced in the MEG protocol. As discussed above, results of our complementary behavioral FTT study suggest that the attenuation of the boost effect in the MEG protocol is likely due to movement repetition immediately before FTT retest, explaining the lack of direct relationship between neurophysiological and behavioral data. Therefore, in addition to the association between age and changes in alpha power rebound, we propose that prior motor sequence learning enhances post-movement mu rhythms rebound during a simple motor task in young participants, a post-training modulatory effect that disappear with age. A lack of increased rebound following learning in older participants is consistent with evidence for diminished inhibitory responses to rTMS applied over M1 in older adults (Todd et al., 2010), suggesting reduced LTD synaptic changes in the aging brain. Likewise, tactile afferent stimulation reduces intracortical inhibition in M1 in young but not in older participants (Smith et al., 2011). According to the authors, this loss of modulation may lead to a decline of fine movement control and practice-induced plasticity. In our MEG study, learning did not modulate the post-movement rebound in the older group. However, rebounds in the alpha and beta bands were as high as the postlearning rebound of young subjects in both SMT sessions. It suggests that a stronger motor inhibitory control might be necessary in aging during the execution of the same motor task than in young subjects. Accordingly, prior studies have reported greater alpha and beta power in sensorimotor regions with aging during the execution of a motor task (Sailer et al., 2000), associated with greater activation in some regions or with the recruitment of additional areas allowing to perform as well as young subjects, e.g. cerebellum, premotor area, prefrontal cortex, caudate nucleus (Sailer et al., 2000; Wu and Hallett, 2005). Recent studies showed that both beta ERD and ERS are sensitive to developmental changes. Children exhibit less post-movement beta rebound than adults, suggesting that this reduced motor inhibition might facilitate motor plasticity and learning in children (Gaetz et al., 2010). More recently, Rossiter et al. (2014) evidenced a higher beta ERD during movement in older participants compared to young adults. According to the authors, this enhanced ERD reflects increased intracortical inhibition which may reduce task-dependent plasticity in aging. In line with these studies, we assume that the higher baseline cortical inhibition for the alpha band in older adults in comparison to the young adults $(t(27)=-2.098, p<0.05)$ might prevent the modulation of the rebound following motor learning in older adults.

Finally, we found that differences in the amplitude of the rebound between groups were significant in the alpha but not in the beta frequency. Dissociation of source locations as well as the different timing of the rebounds for mu-alpha and mu-beta suggest that they may involve distinct functional networks (Hari and Salmelin, 1997). In line with this study, our source reconstruction indicates that the mu-alpha is localized in the postcentral gyrus (i.e. somatosensory cortex) and the mu-beta in the precentral gyrus (i.e. motor cortex). This suggests that the mu-alpha is responsible for somatosensory processes and the mu-beta for motor functions. In the present study, the two motor tasks (SMT and FTT) featured a tactile stimulation of the fingertips (while pressing buttons with the left fingers). Sensory inputs are important to optimize motor control (Smith et al., 2011), and cortical reorganization in the somatosensory cortex can take place following training. Indeed, the representation of left hand fingers is larger in string players 
than in controls, and more pronounced in people who began to play younger (Elbert et al., 1995). Therefore, we surmise that previous motor sequence training may have modified fingers bottom-up somatosensory information processing in young participants, possibly contributing to the boost of performance.

\section{Conclusions}

To sum up, the present study confirms the reproducibility of the boost effect, and evidences using MEG a post-learning modulation in SM1, in agreement with the proposal that the boost represents a "temporary activated state of motor memory" (Hotermans et al., 2006) that can modulate mu rhythms during the execution of simple movements unrelated to the previously learned motor sequence. Moreover, we found evidence for larger cortical reorganization following learning in young than in older participants. Altogether, our findings are consistent with the hypothesis of an age-related decline in the capacity of SM1 to undergo dynamic plastic changes (e.g., Freitas et al., 2011; Rogasch et al., 2009; Smith et al., 2011; Todd et al., 2010).

\section{Acknowledgements}

This research project was financially supported by the Belgian Fonds National de la Recherche Scientifique (FRS-FNRS grant reference 7020836), and the ULB Action de Recherche Concertée (ARC) grant "Pathophysiology of Brain Plasticity Processes in Memory Consolidation". AM is a FRS-FNRS Research Fellow, MB a FRS-FNRS Postdoctoral Researcher, XdT a FRS-FNRS Postdoctoral Specialist, and RL is supported by a "Brains Back to Brussels" grant.

\section{References}

Albouy, G., Ruby, P., Phillips, C., Luxen, A., Peigneux, P., Maquet, P., 2006. Implicit oculomotor sequence learning in humans: time course of offline processing. Brain Res. 1090 (1), 163-171. http://dx.doi.org/10.1016/j.brainres.2006.03.076.

Andres, F.G., Mima, T., Schulman, A.E., Dichgans, J., Hallett, M., Gerloff, C., 1999. Functiona coupling of human cortical sensorimotor areas during bimanual skill acquisition. Brain 122 (0006-8950), 855-870.

Beck, A.T., Rial, W.Y., Rickels, K., 1974. Short form of depression inventory: crossvalidation. Psychol. Rep. 34 (3), 1184-1186.

Bo, J., Seidler, R.D., 2009. Visuospatial working memory capacity predicts the organization of acquired explicit motor sequences. J. Neurophysiol. 101 (6), 3116-3125. http://dx. doi.org/10.1152/jn.00006.2009.

Bo, J., Borza, V., Seidler, R.D., 2009. Age-related declines in visuospatial working memory correlate with deficits in explicit motor sequence learning. Journal of Neurophysiology 102 (5), 2744-2754. http://dx.doi.org/10.1152/jn.00393.2009.

Boonstra, T.W., Daffertshofer, A., Breakspear, M., Beek, P.J., 2007. Multivariate timefrequency analysis of electromagnetic brain activity during bimanual motor learning Neurolmage 36 (2), 370-377. http://dx.doi.org/10.1016/j.neuroimage.2007.03.012.

Brown, R., Robertson, E., Press, D., 2009. Sequence skill acquisition and off-line learning in normal aging. PLoS One 4 (8), e6683.

Bruchon-Schweitzer, M., Paulhan, I., 1990. Manuel de l'inventaire d'Anxiété trait-état (forme Y) Laboratoire de psychologie de la santé, Université de Bordeaux II, France.

Buysse, D.J., Reynolds, C.F., Monk, T.H., Berman, S.R., Kupfer, D.J., 1989. The Pittsburgh Sleep Quality Index: a new instrument for psychiatric practice and research. Psychiatry Res. 28 (2), 193-213.

Caetano, G., Jousmäki, V., Hari, R., 2007. Actor's and observer's primary motor cortices stabilize similarly after seen or heard motor actions. Proc. Natl. Acad. Sci. 104 (21), 9058-9062. http://dx.doi.org/10.1073/pnas.0702453104.

Cassim, F., Szurhaj, W., Sediri, H., Devos, D., Bourriez, J.L., Poirot, I., Guieu, J.D., 2000. Brief and sustained movements: differences in event-related (de)synchronization (ERD/ ERS) patterns. Clin. Neurophysiol. 111, 2032-2039.

Censor, N., Dimyan, M.A., Cohen, L.G., 2010. Modification of existing human motor memories is enabled by primary cortical processing during memory reactivation. Curr. Biol. 20 (17), 1545-1549. http://dx.doi.org/10.1016/j.cub.2010.07.047.

Chatrian, G.E., Petersen, M.C., Lazarte, J.A., 1959. The blocking of the rolandic wicket rhythm and some central changes related to movement. Electroencephalogr. Clin. Neurophysiol. 11 (3), 497-510. http://dx.doi.org/10.1016/0013-4694(59)90048-3.

Cheyne, D.O., 2012. MEG studies of sensorimotor rhythms: a review. Exp. Neurol. http:// dx.doi.org/10.1016/j.expneurol.2012.08.030.

Cheyne, D., Gaetz, W., Garnero, L., Lachaux, J.-P., Ducorps, A., Schwartz, D., Varela, F.J., 2003. Neuromagnetic imaging of cortical oscillations accompanying tactile stimulation. Cogn. Brain Res. 17 (3), 599-611. http://dx.doi.org/10.1016/S0926-6410(03)00173-3.

Cleeremans, A., McClelland, J.L., 1991. Learning the structure of event sequences. J. Exp. Psychol. Gen. 120 (3), 235-253.
Collet, L., Cottraux, J., 1986. Inventaire abrégé de la dépression de Beck (13 items): Étude de la validité concurrente avec les échelles de Hamilton et de ralentissement de Widlöcher (The shortened Beck Depression Inventory: Study of the concurrent validity with the Hamilton Depression Rating Scale and the Widlöcher retardation Rating Scale). L'Encéphale 12 (2), 77-79.

Cooke, S.F., Bliss, T.V.P., 2006. Plasticity in the human central nervous system. Brain 129 (7), 1659-1673. http://dx.doi.org/10.1093/brain/awl082.

Dale, A.M., Sereno, M.I., 1993. Improved localization of cortical activity by combining EEG and MEG with MRI cortical surface reconstruction: a linear approach. J. Cogn. Neurosci. 5 (2), 162-176. http://dx.doi.org/10.1162/jocn.1993.5.2.162.

Dirnberger, G., Duregger, C., Lindinger, G., Lang, W., 2004. Habituation in a simple repetitive motor task: a study with movement-related cortical potentials. Clin. Neurophysiol. 115 (2), 378-384. http://dx.doi.org/10.1016/S1388-2457(03)00328-6.

Doyon, J., Bellec, P., Amsel, R., Penhune, V., Monchi, O., Carrier, J., Benali, H., 2009. Contributions of the basal ganglia and functionally related brain structures to motor learning. Behav. Brain Res. 199 (1), 61-75. http://dx.doi.org/10.1016/j.bbr.2008.11.012.

Dudai, Y., 2004. The neurobiology of consolidations, or, how stable is the engram? Annu. Rev. Psychol. 55, 51-86.

Elbert, T., Pantev, C., Wienbruch, C., Rockstroh, B., Taub, E., 1995. Increased cortical representation of the fingers of the left hand in string players. Science 270 (5234), 305-307. http://dx.doi.org/10.1126/science.270.5234.305.

Ellis, B.W., Johns, M.W., Lancaster, R., Raptopoulos, P., Angelopoulos, N., Priest, R.G. 1981. The St. Mary's Hospital sleep questionnaire: a study of reliability. Sleep 4 (1), 93-97

Engel, A.K., Fries, P., 2010. Beta-band oscillations - signalling the status quo? Curr. Opin. Neurobiol. 20 (2), 156-165. http://dx.doi.org/10.1016/j.conb.2010.02.015.

Erbil, N., Ungan, P., 2007. Changes in the alpha and beta amplitudes of the central EEG during the onset, continuation, and offset of long-duration repetitive hand movements. Brain Res. 1169, 44-56. http://dx.doi.org/10.1016/j.brainres.2007.07.014.

Francis, K.L., Spirduso, W.W., 2000. Age differences in the expression of manual asymmetry. Exp. Aging Res. 26 (2), 169-180. http://dx.doi.org/10.1080/036107300243632.

Frankland, P.W., Bontempi, B., 2005. The organization of recent and remote memories. Nat. Rev. Neurosci. 6, 119-130.

Freitas, C., Perez, J., Knobel, M., Tormos, J.M., Oberman, L., Eldaief, M., Pascual-Leone, A., 2011. Changes in cortical plasticity across the lifespan. Front. Aging Neurosci. 3. http://dx.doi.org/10.3389/fnagi.2011.00005.

Freyer, F., Becker, R., Dinse, H.R., Ritter, P., 2013. State-dependent perceptual learning. J. Neurosci. 33 (7), 2900-2907.

Gaetz, W., Macdonald, M., Cheyne, D., Snead, O.C., 2010. Neuromagnetic imaging of movement-related cortical oscillations in children and adults: age predicts postmovement beta rebound. Neurolmage 51 (2), 792-807. http://dx.doi.org/10.1016/j. neuroimage.2010.01.077.

Hall, C.R., Martin, K.A. 1997. Measuring movement imagery abilities: A revision of the Movement Imagery Questionnaire. Journal of Mental Imagery 21 (1-2), 143-154.

Hari, R., 2006. Action-perception connection and the cortical mu rhythm. Prog. Brain Res. $159,253-260$.

Hari, R., Salmelin, R., 1997. Human cortical oscillations: a neuromagnetic view through the skull. Trends Neurosci. 20 (1), 44-49. http://dx.doi.org/10.1016/S0166-2236(96) 10065-5.

Horne, J.A., Ostberg, O., 1976. A self-assessment questionnaire to determine morningnesseveningness in human circadian rhythms. Int. J. Chronobiol. 4 (2), 97-110.

Hotermans, C., Peigneux, P., de Noordhout, A.M., Moonen, G., Maquet, P., 2006. Early boost and slow consolidation in motor skill learning. Learn. Mem. 13 (5), 580-583.

Hotermans, C., Peigneux, P., de Noordhout, A.M., Moonen, G., Maquet, P., 2008. Repetitive transcranial magnetic stimulation over the primary motor cortex disrupts early boost but not delayed gains in performance in motor sequence learning. Eur. J. Neurosci. 28 (6), 1216-1221.

Jones, S.R., Pritchett, D.L., Sikora, M.A., Stufflebeam, S.M., Hämäläinen, M., Moore, C.I., 2009. Quantitative analysis and biophysically realistic neural modeling of the MEG Mu rhythm: rhythmogenesis and modulation of sensory-evoked responses. J. Neurophysiol. 102 (6), 3554-3572. http://dx.doi.org/10.1152/jn.00535.2009.

Jurkiewicz, M.T., Gaetz, W.C., Bostan, A.C., Cheyne, D., 2006. Post-movement beta rebound is generated in motor cortex: evidence from neuromagnetic recordings. Neurolmage 32 (3), 1281-1289. http://dx.doi.org/10.1016/j.neuroimage.2006.06.005.

Karni, A., Meyer, G., Jezzard, P., Adams, M.M., Turner, R., Ungerleider, L.G., 1995. Functional MRI evidence for adult motor cortex plasticity during motor skill learning. Nature 377 (6545), 155-158.

Kim, J.S., Chung, C.K., 2007. Robust source analysis of oscillatory motor cortex activity with inherently variable phase delay. NeuroImage 37 (2), 518-529. http://dx.doi. org/10.1016/j.neuroimage.2007.04.068

Klimesch, W., Sauseng, P., Hanslmayr, S., 2007. EEG alpha oscillations: the inhibitiontiming hypothesis. Brain Res. Rev. 53 (1), 63-88. http://dx.doi.org/10.1016/j. brainresrev.2006.06.003.

Lupien, S.J., Wan, N., 2004. Successful ageing: from cell to self. Philos. Trans. R. Soc. Lond. B Biol. Sci. 359 (1449), 1413-1426.

Malleret, G., Alarcon, J.M., Martel, G., Takizawa, S., Vronskaya, S., Yin, D., Shumyatsky, G.P., 2010. Bidirectional regulation of hippocampal long-term synaptic plasticity and its influence on opposing forms of memory. J. Neurosci. 30 (10), 3813-3825.

Mattis, S., 1976. Mental status examination for organic mental syndrome in the elderly patient. Geriatric Psychiatry. Grune and Stratton, New York, pp. 77-121.

McAllister, C.J., Rönnqvist, K.C., Stanford, I.M., Woodhall, G.L., Furlong, P.L., Hall, S.D., 2013. Oscillatory beta activity mediates neuroplastic effects of motor cortex stimulation in humans. J. Neurosci. 33 (18), 7919-7927.

McGaugh, J.L., 2000. Memory - a century of consolidation. Science 287, 248-251.

Muellbacher, W., Ziemann, U., Wissel, J., Dang, N., Kofler, M., Facchini, S., Hallett, M., 2002. Early consolidation in human primary motor cortex. Nature 415 (6872), 640-644. 
Nasreddine, Z.S., Phillips, N.A., Bédirian, V., Charbonneau, S., Whitehead, V., Collin, I. Chertkow, H., 2005. The Montreal Cognitive Assessment, MoCA: a brief screening tool for mild cognitive impairment. J. Am. Geriatr. Soc. 53 (4), 695-699. http://dx. doi.org/10.1111/j.1532-5415.2005.53221.x.

Neuper, C., Wörtz, M., Pfurtscheller, G., 2006. ERD/ERS patterns reflecting sensorimotor activation and deactivation. Prog. Brain Res. 159, 211-222.

Nichols, T.E., Holmes, A.P., 2002. Nonparametric permutation tests for functional neuroimaging: a primer with examples. Hum. Brain Mapp. 15 (1), 1-25. http://dx.doi.org/ 10.1002/hbm.1058.

Noh, N.A., Fuggetta, G., Manganotti, P., Fiaschi, A., 2012. Long lasting modulation of cortical oscillations after continuous Theta Burst Transcranial Magnetic Stimulation. PLoS One 7 (4), e35080. http://dx.doi.org/10.1371/journal.pone.0035080.

Oldfield, R.C., 1971. The assessment and analysis of handedness: the Edinburgh inventory. Neuropsychologia 9 (1), 97-113.

Orban, P., Peigneux, P., Lungu, O., Debas, K., Barakat, M., Bellec, P., Doyon, J., 2011. Functional neuroanatomy associated with the expression of distinct movement kinematics in motor sequence learning. Neuroscience 179, 94-103. http://dx.doi.org/10. 1016/j.neuroscience.2011.01.040.

Peigneux, P., Orban, P., Balteau, E., Degueldre, C., Luxen, A., Laureys, S., Maquet, P., 2006. Offline persistence of memory-related cerebral activity during active wakefulness. PLoS Biol. 4 (4), e100.

Penhune, V.B. Steele, C.J., 2012. Parallel contributions of cerebellar, striatal and M1 mechanisms to motor sequence learning. Behav. Brain Res. 226 (2), 579-591. http://dx.doi. org/10.1016/j.bbr.2011.09.044

Pfurtscheller, G., Lopes da Silva, F.H., 1999. Event-related EEG/MEG synchronization and desynchronization: basic principles. Clin. Neurophysiol. 110 (11), 1842-1857. http://dx.doi.org/10.1016/S1388-2457(99)00141-8.

Pfurtscheller, G., Stancák Jr., A., Neuper, C., 1996. Post-movement beta synchronization. A correlate of an idling motor area? Electroencephalogr. Clin. Neurophysiol. 98 (4), 281-293. http://dx.doi.org/10.1016/0013-4694(95)00258-8.

Pineda, J.A., 2005. The functional significance of mu rhythms: translating "seeing" and "hearing" into "doing". Brain Res. Rev. 50, 57-68.

Press, D.Z., Casement, M.D., Pascual-Leone, A., Robertson, E.M., 2005. The time course of off-line motor sequence learning. Cogn. Brain Res. 25 (1), 375-378.

Ritter, P., Moosmann, M., Villringer, A., 2009. Rolandic alpha and beta EEG rhythms' strengths are inversely related to fMRI-BOLD signal in primary somatosensory and motor cortex. Hum. Brain Mapp. 30, 1168-1187.

Robertson, E.M., Press, D.Z., Pascual-Leone, A., 2005. Off-line learning and the primary motor cortex. J. Neurosci. 25 (27), 6372-6378.

Rogasch, N.C., Dartnall, T.J., Cirillo, J., Nordstrom, M.A., Semmler, J.G., 2009. Corticomotor plasticity and learning of a ballistic thumb training task are diminished in older adults. J. Appl. Physiol. 107 (6), 1874-1883. http://dx.doi.org/10.1152/japplphysiol.00443.2009.

Rossiter, H.E., Davis, E.M., Clark, E.V., Boudrias, M.-H., Ward, N.S., 2014. Beta oscillations reflect changes in motor cortex inhibition in healthy ageing. Neurolmage 91 , 360-365. http://dx.doi.org/10.1016/j.neuroimage.2014.01.012.

Sailer, A., Dichgans, J., Gerloff, C., 2000. The influence of normal aging on the cortical processing of a simple motor task. Neurology 55 (7), 979-985.
Salmelin, R., Hámáaláinen, M. Kajola, M., Hari, R., 1995. Functional segregation of movement-related rhythmic activity in the human brain. NeuroImage 2 (4), 237-243. http://dx.doi.org/10.1006/nimg.1995.1031.

Sawaki, L., Yaseen, Z., Kopylev, L., Cohen, L.G., 2003. Age-dependent changes in the ability to encode a novel elementary motor memory. Ann. Neurol. 53 (4), 521-524. http:// dx.doi.org/10.1002/ana.10529.

Schmitz, R., Schabus, M., Perrin, F., Luxen, A., Maquet, P., Peigneux, P., 2009. Recurrent boosting effects of short inactivity delays on performance: an ERPs study. BMC Res. Notes 2 (170), 1-8.

Schuch, S., Bayliss, A., Klein, C., Tipper, S., 2010. Attention modulates motor system activation during action observation: evidence for inhibitory rebound. Exp. Brain Res. 205 (2), 235-249. http://dx.doi.org/10.1007/s00221-010-2358-4.

Sheikh, J.I., Yesavage, J.A., 1986. Geriatric Depression Scale (GDS). Recent evidence and development of a shorter version. Clin. Gerontol. 5 (1-2), 165-173. http://dx.doi org/10.1300/J018v05n01_09.

Smith, A.E., Ridding, M.C., Higgins, R.D., Wittert, G.A., Pitcher, J.B., 2011. Cutaneous afferent input does not modulate motor intracortical inhibition in ageing men. Eur. J. Neurosci. 34 (9), 1461-1469. http://dx.doi.org/10.1111/j.1460-9568.2011.07869.x.

Spencer, R.M.C., Gouw, A.M., Ivry, R.B., 2007. Age-related decline of sleep-dependent consolidation. Learn. Mem. 14 (7), 480-484.

Tallon-Baudry, C., Bertrand, O., Delpuech, C., Pernier, J., 1996. Stimulus specificity of phase-locked and non-phase-locked $40 \mathrm{~Hz}$ visual responses in human. J. Neurosci. Off. J. Soc. Neurosci. 16 (13), 4240-4249.

Taulu, S., Simola, J., Kajola, M., 2005. Applications of the signal space separation method. IEEE Trans. Signal Process. 53 (9), 3359-3372. http://dx.doi.org/10.1109/TSP.2005. 853302.

Todd, G., Kimber, T.E., Ridding, M.C., Semmler, J.G., 2010. Reduced motor cortex plasticity following inhibitory rTMS in older adults. Clin. Neurophysiol. 121 (3), 441-447.

Verwey, W.B., 2010. Diminished motor skill development in elderly: indications for limited motor chunk use. Acta Psychol. 134 (2), 206-214. http://dx.doi.org/10. 1016/j.actpsy.2010.02.001.

Walker, M.P., 2005. A refined model of sleep and the time course of memory formation. Behav. Brain Sci. 28 (1), 51-104.

Walker, M.P., Stickgold, R., 2006. Sleep, memory, and plasticity. Annu. Rev. Psychol. 57, 139-166.

Wilson, J.K., Baran, B., Pace-Schott, E.F., Ivry, R.B., Spencer, R.M.C., 2012. Sleep modulates word-pair learning but not motor sequence learning in healthy older adults. Neurobiol. Aging 33 (5), 991-1000. http://dx.doi.org/10.1016/j.neurobiolaging.2011. 06.029.

Wu, T., Hallett, M., 2005. The influence of normal human ageing on automatic movements. J. Physiol. 562 (2), 605-615. http://dx.doi.org/10.1113/jphysiol.2004.076042.

Zhuang, P., Toro, C., Grafman, J., Manganotti, P., Leocani, L., Hallett, M., 1997. Event-related desynchronization (ERD) in the alpha frequency during development of implicit and explicit learning. Electroencephalogr. Clin. Neurophysiol. 102 (4), 374-381.

Ziemann, U., Iliać, T.V., Pauli, C., Meintzschel, F., Ruge, D., 2004. Learning modifies subsequent induction of long-term potentiation-like and long-term depression-like plasticity in human motor cortex. J. Neurosci. 24 (7), 1666-1672. 